$\begin{array}{cl}\begin{array}{c}\text { Revue } \\ \text { de /histoire }\end{array} & \text { Revue de l'histoire des religions } \\ \text { des religions } & \begin{array}{l}2 \mid \mathbf{2 0 1 0} \\ \text { Varia }\end{array}\end{array}$

\title{
Définir les « cathares » : le dualisme dans les registres d'inquisition
}

Defining «Cathars ": Dualism through inquisitional registers

David Zbíral

\section{(2) OpenEdition}

\section{Journals}

Édition électronique

URL : http://journals.openedition.org/rhr/7575

DOI : $10.4000 /$ rhr.7575

ISSN : 2105-2573

Éditeur

Armand Colin

Édition imprimée

Date de publication : 1 juin 2010

Pagination : 195-210

ISBN : 978-2200-92656-4

ISSN : 0035-1423

Référence électronique

David Zbíral, « Définir les « cathares » : le dualisme dans les registres d'inquisition », Revue de l'histoire des religions [En ligne], 2 | 2010, mis en ligne le 01 juin 2013, consulté le 02 mai 2019. URL : http:// journals.openedition.org/rhr/7575; DOI : 10.4000/rhr.7575 


\section{Définir les « cathares " : le dualisme dans les registres d'inquisition}

Dans la discussion scientifique de ces dernières années, beaucoup d'idées sur ce qu'on appelle le " catharisme » ont été revisitées, sans que le raisonnement ait toujours été mené à terme. Il nous semble logique de commencer l'analyse critique de ce terme par une révision des concepts sous-jacents qui le définissent. Le dualisme en est un, que les "registres d'inquisition " permettent de scruter. On trouve dans cette source des propos dualistes qui ne s'inspirent pas de la prédication des bons hommes (ou "parfaits cathares ») et qui ne sont pas compatibles avec l'idée polémique et inquisitoriale de l' "hérésie manichéenne ". Il semble donc que le terme de dualisme, sans autres précisions, est trop vague et insuffisant pour rendre compte du concept du catharisme.

\section{Defining « Cathars » : Dualism through inquisitional registers}

Many notions concerning "Catharism» have been revisited in the scholarly debate of recent years, although the reasoning in question has not always been brought to fruition. It seems logical to begin a critical analysis of this term by reviewing the underlying concepts that define it. Among these is dualism, which may be examined through "inquisitional registers ». These sources contain dualist statements that are not inspired by the preaching of the Good Men (or "Cathar Perfects") and are not compatible with the polemical and inquisitorial idea of "Manichean heresy ". It seems therefore that dualism, too vague a term, is insufficient to render the concept of Catharism without further specifications. 


\section{INTRODUCTION}

Le catharisme a longtemps été un concept presque incontesté. Même les chercheurs qui soulignaient la corrélation entre l'hérésie et les transformations de l'orthodoxie, l'importance des contextes locaux et le caractère construit de l'hérésie ont gardé l'idée d'un mouvement cathare plutôt homogène - sinon même une Église alternative - issu de missions bogomiles en Occident ${ }^{1}$.

Or l'existence du mouvement bogomilo-cathare qui se serait manifesté dans une bonne moitié de l'Europe entre le $x^{e}$ et le $\mathrm{XV}^{\mathrm{e}}$ siècle s'est montrée moins avérée qu'on n'était habitué à le penser. Ce sont d'abord des chercheurs italiens qui se sont mis à relativiser ce concept. Dans son Medioevo cristiano, Raffaello Morghen évoquait des «manifestations de la prétendue hérésie cathare, dispersées, sans relations évidentes entre elles, dans des pays les plus divers de l'Europe à travers deux siècles ${ }^{2} »$. Gabriele Zanella, lui, soulignait certains problèmes des « hiérarchies cathares » supposées par Antoine Dondaine ${ }^{3}$ pour y substituer un modèle de l'hérésie lombarde à caractère plutôt indistinct et « itinérant $t^{4} »$. Fait remarquable, ces chercheurs n'ont eu d'abord qu'une très faible influence sur la recherche internationale menée sur les cathares, constituée principalement par les articles du P. Dondaine et par les grandes synthèses de Jean Duvernoy5. Même l'article de vulgarisation de Jean-Louis

1. On pense notamment à l'exemple de Robert I. Moore (Robert I. Moore, The Formation of a Persecuting Society. Power and Deviance in Western Europe, 950-1250, Oxford, 1987; id., The Origins of European Dissent, Toronto, 1994, p. 168-240).

2. Raffaello Morghen, Medioevo cristiano, Roma, 1991 (1 ${ }^{\text {re }}$ éd. 1951), p. 193.

3. Antoine Dondaine, "La hiérarchie cathare en Italie I. Le De heresi catharorum », Archivum Fratrum Praedicatorum, 19, 1949, p. 280-312; id., " La hiérarchie cathare en Italie II. Le Tractatus de hereticis d'Anselme d'Alexandrie », Archivum Fratrum Praedicatorum, 20, 1950, p. 234-324.

4. Gabriele Zanella, Itinerari ereticali. Patari e Catari tra Rimini e Verona, Roma, 1986; id., « Malessere ereticale in Valle Padana (1260-1308) », Rivista di storia e letteratura religiosa, 14, n ${ }^{\circ} 3,1978$, p. 341-390, repris dans id., Hereticalia. Temi e discussioni, Spoleto, 1995, p. 15-66.

5. Jean Duvernoy, Le Catharisme, vol. 1, La Religion des cathares, Toulouse, 1976; id., Le Catharisme, vol. 2, L'Histoire des cathares, Toulouse, 1979. 
Biget «Les Cathares. Mise à mort d'une légende », paru en $1986^{6}$, n'a changé en rien cette tendance; il faut d'ailleurs noter que s'il dénonce avec vigueur quelques idées fausses (comme celle du «Languedoc - pays cathare »), il ne met pour autant pas en cause la réalité du catharisme en tant que telle. Quelques années plus tard, Monique Zerner publiait un article intitulé « Du court moment où on appela les hérétiques des Bougres ${ }^{7} »$, qui n'a cependant pas suscité l'attention qu'il aurait méritée. Dans une discussion écrite avec Anne Brenon, Jean-Louis Biget a préfiguré beaucoup de thèmes aujourd'hui courants ${ }^{8}$. Le volume Inventer l'hérésie? Discours polémiques et pouvoirs avant l'Inquisition, publié sous la direction de Monique Zerner' , a marqué une nouvelle direction dans l'historiographie en réorientant l'intérêt scientifique vers les mécanismes de la construction de l'hérésie dans le discours polémique. Or la contribution très documentée de Jean-Louis Biget parue dans ce volume ${ }^{10}$ ne se concentre pas sur le concept du catharisme en tant que tel, mais plutôt sur l'importance du contexte local. Il a fallu attendre la parution, en 2001, de L'Histoire du catharisme en discussion. Le " concile " de Saint-Félix (1167) ${ }^{11}$, où l'authenticité d'une source clé connue sous le nom de «Charte de Niquinta » se voit contestée, pour que le débat se généralise et devienne plus vivant, parfois même pointu ${ }^{12}$.

6. Jean-Louis Biget, « Les Cathares. Mise à mort d'une légende », L'Histoire, 94, 1986, p. 10-21.

7. Monique Zerner, «Du court moment où on appela les hérétiques des « Bougres », et quelques déductions », Cahiers de civilisation médiévale, 32, $\mathrm{n}^{\circ} 4$, 1989, p. 305-324.

8. Voir la version complète, «Réponse de Jean-Louis Biget, à la demande de la direction de l'Histoire », Heresis, 24, 1995, p. 94-99.

9. Monique Zerner (dir.), Inventer l'hérésie? Discours polémiques et pouvoirs avant l'Inquisition, Nice, 1998.

10. J.-L. Biget, « « Les Albigeois » : remarques sur une dénomination », dans M. Zerner (dir.), Inventer l'hérésie?, p. 219-255.

11. M. Zerner (dir.), L'Histoire du catharisme en discussion. Le " concile» de Saint-Félix (1167), Nice, 2001.

12. Julien Roche, "Enjeux et embûches de la recherche actuelle sur le catharisme : l'exemple de la charte de Nicetas ", dans Emmanuel Le Roy Ladurie (dir.), Autour de Montaillou, un village occitan. Histoire et religiosité d'une communauté villageoise au Moyen Âge, Castelnaud la Chapelle, 2001, p. 249-265; $i d$., «La Charte de Niquinta : un point sur la controverse », dans Edina Bozóky (dir.), Bogomiles, Patarins et Cathares, Slavica Occitania, 16, 2003, p. 229-245; Daniele Solvi, «L'histoire du catharisme en discussion. Le « concile» de SaintFélix (1167)», Studi medievali, 45, n 2, 2004, p. 815-828; Francesco Zambon, «Où en est le problème des Actes du « concile» de Saint-Félix? À propos de «L'histoire du catharisme en discussion»", dans Martin Aurell (dir.), Les 
En 2001 également a paru un article pénétrant de Mark G. Pegg qui résume d'une manière très lucide certains problèmes du concept des « cathares ${ }^{13}$ ». Non moins important est l'apport de Julien Théry, qui a contesté ouvertement le concept du catharisme ${ }^{14}$. Pilar Jiménez Sanchez a publié des articles où elle ne va pas jusqu'à mettre en cause l'existence d'un mouvement cathare européen, mais où elle choisit de porter plus d'attention qu'auparavant aux différences des groupes dits « cathares » et préfère le concept des catharismes au pluriel au concept traditionnel du catharisme ${ }^{15}$. Un volume publié sous la direction de Caterina Bruschi et Peter Biller a réaffirmé l'importance des textes dans la définition et la répression de l'hérésie ${ }^{16}$. En 2003, JeanLouis Biget a souligné de nouveau l'importance des contextes locaux en rejetant explicitement cette fois-ci l'origine bogomile des prétendus cathares ${ }^{17}$. En 2006, dans l'ouvrage majeur Des contestataires aux «cathares $»^{18}$, Uwe Brunn a renouvelé complètement l'historiographie de l'hérésie en Rhénanie : il a mis en évidence la faiblesse de l'hypothèse de l'origine orientale des dissidents rhénans et a mon-

Cathares devant l'Histoire. Mélanges offerts à Jean Duvernoy, Cahors, 2005, p. 135-144; David Zbíral, « La Charte de Niquinta. Un faux moderne? », Heresis, 42-43, 2005, p. 139-159; M. Zerner, « Mise au point sur Les cathares devant l'histoire et retour sur L'histoire du catharisme en discussion. Le débat sur la Charte de Niquinta n'est pas clos ", Journal des savants, 2006, n² 2, p. 253-273; D. Zbíral, «La Charte de Niquinta et les récits sur les commencements des églises cathares en Italie et dans le Midi », Heresis, 44-45, 2006, p. 135-162.

13. Mark G. Pegg, " On Cathars, Albigenses and Good Men of Languedoc. Historiographical Essay », Journal of Medieval History, 27, 2001, p. 181-195.

14. Julien Théry, «L'hérésie des bons hommes. Comment nommer la dissidence religieuse non vaudoise ni béguine en Languedoc (XII ${ }^{\mathrm{e}}$-début du XIV ${ }^{\mathrm{e}}$ siècle)? », Heresis, 36-37, 2002, p. 75-117.

15. Pilar Jiménez Sanchez, « Catharisme ou catharismes? Variations spatiales et temporelles dans l'organisation et dans l'encadrement des communautés dites " cathares » », Heresis, 39, 2003, p. 35-61 ; ead., " Variations dans les rites sacramentaires des cathares. L'exemple de l'initiation chrétienne (XII $\mathrm{e}-\mathrm{XIII}{ }^{\mathrm{e}}$ siècles) », Bollettino della Società di studi valdesi, 121, 2004, p. 37-58. Pilar Jiménez Sanchez complète son raisonnement dans sa récente monographie Les catharismes. Modèles dissidents du christianisme médiéval (XII ${ }^{e}-X I I I^{e}$ siècles), Rennes, 2008.

16. Caterina Bruschi, Peter Biller (dir.), Texts and the Repression of Medieval Heresy, York, 2003.

17. J.-L. Biget, "Les bons hommes sont-ils les fils des bogomiles? Examen critique d'une idée reçue ", dans E. Bozóky (dir.), Bogomiles, Patarins et Cathares, Slavica Occitania, 16, 2003, p. 133-188, repris dans J.-L. Biget, Hérésie et inquisition dans le Midi de la France, Paris, 2007, p. 63-105.

18. Uwe Brunn, Des contestataires aux «cathares ». Discours de réforme et propagande antihérétique dans les pays du Rhin et de la Meuse avant l'Inquisition, Paris, 2006. 
tré que les cathari sont nés dans le monde intellectuel d'Eckbert de Schönau qui a exhumé ce mot de la tradition hérésiologique pour définir, dans les années 1150-1160, les dissidents contemporains en Rhénanie, d'où cette appellation s'est ensuite diffusée.

On assiste donc à un changement de perspective important. Certes, on est en droit de se demander si certains jugements qui veulent trop vite mettre à mort le catharisme ne dépassent pas les recherches qu'elles prétendent conclure. Mais il n'y a pas lieu de nier que le «catharisme » est un concept dont l'héritage est tellement lourd que son analyse critique s'impose.

Il est tout naturel qu'une telle analyse critique parte d'un examen des idées sur lesquelles ce concept repose, dont deux semblent centrales : le consolament (baptême dissident par l'imposition des mains) et le dualisme. Une telle analyse est d'autant plus importante que ces concepts sous-jacents restent assez flous et mal définis dans la plupart des travaux historiques - trop en tout cas pour pouvoir établir d'une manière convaincante une filiation de tant de groupes un peu partout en Europe à travers cinq siècles.

Dans un livre publié en $2007^{19}$, j'ai essayé d'examiner le deuxième de ces concepts sous-jacents - le dualisme. Celui-ci a été identifié comme la substance de l'hérésie cathare dans le tout premier écrit à employer le terme cathari pour désigner des hérétiques médiévaux, le Liber contra hereses katharorum, rédigé entre 1163 et $1165^{20}$ par Eckbert de Schönau, chanoine à Bonn, puis moine bénédictin à Schönau. Cette première définition de l'hérésie cathare par le dualisme ne manque pas d'intérêt, d'autant que tous les passages du Liber qui évoquent le dualisme reprennent bel et bien les polémiques de saint Augustin contre les manichéens, dont les cathari seraient, selon Eckbert, des descendants ${ }^{21}$. Eckbert a utilisé ses connaissances de la tradition hérésiologique à propos

19. D. Zbíral, Největši hereze. Dualismus, učenecká vyprávění o katarství a budováni křestanské Evropy, Praha, 2007.

20. U. Brunn, Des contestataires aux « cathares », p. 218-219.

21. Jacques-Paul Migne (éd.), Patrologia Latina, t. 195, Parisiis, 1855, col. 16, 17 etc. La filiation manichéenne du bogomilisme et du catharisme a été rejetée par l'historiographie moderne comme un mythe polémique : Henri-Charles Puech, André Vaillant, Le Traité contre les Bogomiles de Cosmas le Prêtre. Traduction et étude, Paris, 1945, p. 304-316; J. Duvernoy, Le Catharisme, vol. 1, La Religion des cathares, p. 360; Ylva Hagman, "Le catharisme, un néo-manichéisme? », Heresis, 21, 1993, p. 47-59. 
des catharistae (fraction manichéenne selon saint Augustin) et des cathari pour bâtir son discours sur des dissidents contemporains ${ }^{22}$.

Au lieu d'aborder ici une discussion globale sur le dualisme, je voudrais me pencher sur un point précis : le concept du dualisme ou d' " hérésie manichéenne » dans les registres d'inquisition. Dans cette catégorie de textes, on trouve des propos dualistes qui sont plus ou moins compatibles avec la vision des inquisiteurs, certes, mais aussi d'autres qui ne le sont pas du tout. Mon objectif est d'étudier ces marges du « dualisme » et leur rapport avec la perspective inquisitoriale afin de nuancer le concept du catharisme, dont le dualisme serait à la fois la substance et le trait distinctif.

La source principale de cette enquête sera le registre de Jacques Fournier $^{23}$, évêque de Pamiers (1317-1326), puis évêque de Mirepoix, cardinal et enfin pape sous le nom de Benoît XII ${ }^{24}$. Ce choix est basé sur une recherche préliminaire, qui a identifié ce registre comme le plus riche en matière de «marges » du dualisme. Par le détail des dépositions, qui ont inspiré entre autres la monographie aujourd'hui classique d'Emmanuel Le Roy Ladurie ${ }^{25}$, ce registre offre des possibilités exceptionnelles de confronter la vision inquisitoriale à ces « marges dualistes ».

\section{LE DUALISME DES INQUISITEURS}

Il y a eu beaucoup de discussions à propos de la nature de l'Inquisition médiévale. L'image classique d'une institution toute-puissante

22. U. Brunn, "Cathari, catharistae et cataphrygae, ancêtres des cathares du XII ${ }^{\mathrm{e}}$ siècle? », Heresis, 36-37, 2002, p. 183-200; id., Des contestataires aux " cathares », p. 321-333.

23. Vaticano, Biblioteca Apostolica Vaticana, ms. Vat. Lat. 4030; J. Duvernoy (éd.), Le Registre d'inquisition de Jacques Fournier, évêque de Pamiers (1318-1325), 3 vol., Toulouse, 1965; J. Duvernoy (éd.), Le Registre d'inquisition de Jacques Fournier, évêque de Pamiers (1318-1325). Corrections, Toulouse, 1972; J. Duvernoy (trad.), Le Registre d'inquisition de Jacques Fournier (évêque de Pamiers), 1318-1325, 3 vol., Paris, La Haye, New York, 1977-1978.

24. Pour une présentation plus détaillée, voir Jacques Paul, "L'Inquisition de Carcassonne. Jacques Fournier (1317-1326), un inquisiteur professionnel », dans Laurent Albaret (dir.), Les Inquisiteurs. Portraits de défenseurs de la foi en Languedoc (XIII ${ }^{e}$-XIV siècles), Toulouse, 2001, p. 133-140 et Sylvain Piron, " Un avis retrouvé de Jacques Fournier », Médiévales, 54, 2008, p. 113-134.

25. E. Le Roy Ladurie, Montaillou, village occitan de 1294 à 1324, Paris, 1975. 
et rigidement centralisée a été mise en question, non sans raison, par différents chercheurs ${ }^{26}$. Pour autant, il n'est pas question de nier qu'en général, le fonctionnement de l'officium inquisitionis heretice pravitatis au Moyen Âge avait un fonds commun bien identifiable ${ }^{27}$. Il y a des régularités évidentes en ce qui concerne le cadre juridique, le langage, les procédures, les actes, les manuels des inquisiteurs et leur savoir sur les hérésies. Il existe un discours partagé parmi les inquisiteurs.

Dans ce discours inquisitorial, la question centrale est celle de l'identification, des traits distinctifs des différentes hérésies. Pour les inquisiteurs, un des caractères distinctifs principaux de «l'hérésie cathare » est précisément le dualisme. Divers traités et manuels d'inquisiteurs insistent sur le fait que deux croyances " dualistes ", la croyance en deux dieux et la croyance selon laquelle le monde a été créé par le diable, constituent la substance de l'hérésie cathare ou «manichéenne ${ }^{28} »$. La ressemblance entre l'image de l'hérésie cathare dans le discours inquisitorial et dans le discours scientifique est un premier élément qui incite à la prudence.

26. James B. Given, "The Inquisitors of Languedoc and the Medieval Technology of Power », The American Historical Review, 94, 1989, $\mathrm{n}^{\circ}$ 2, p. 339, 356; Edward Peters, Inquisition, New York, 1988; Jeffrey B. Russell, Dissent and Order in the Middle Ages. The Search for Legitimate Authority, New York, 1992, p. 54-55; Richard Kieckhefer, "The Office of Inquisition and Medieval Heresy. The Transition from Personal to Institutional Jurisdiction », Journal of Ecclesiastical History, 46, 1995, n 1, p. 36-61 ; Mark G. Pegg, The Corruption of Angels. The Great Inquisition of 1245-1246, Princeton, 2001, p. 33; id., « On Cathars, Albigenses and Good Men of Languedoc. Historiographical Essay », p. 192. Pour un précis récent et très documenté de l'Inquisition médiévale, voir Paweł Kras, Ad abolendam diversarum haeresium pravitatem. System inkwizycyjny w średniowiecznej Europie, Lublin, 2006.

27. J. B. Given, « The Inquisitors of Languedoc and the Medieval Technology of Power », p. 339.

28. Thomas Käppeli (éd.), «Une somme contre les hérétiques de S. Pierre Martyr (?) », Archivum Fratrum Praedicatorum, 17, 1947, p. 320; Franjo Šanjek (éd.), «Raynerius Sacconi O. P. Summa de Catharis», Archivum Fratrum Praedicatorum, 44, 1974, p. 42; A. Dondaine (éd.), "La hiérarchie cathare en Italie II. Le Tractatus de hereticis d'Anselme d'Alexandrie », p. 312, 319-320; Francesca Lomastro Tognato éd., «Un manuale inquisitoriale vicentino: Le Constitutiones sacre inquisitionis », dans ead., L'eresia a Vicenza nel Duecento. Dati, problemi e fonti, Vicenza, 1988, p. 178; Guillaume Mollat (éd.), Bernard Gui, Manuel de l'inquisiteur, vol. 1, Paris, 1926, p. 10. A. Dondaine, « Le manuel de l'inquisiteur (1230-1330) », Archivum Fratrum Praedicatorum, 17, 1947, p. 85-194 reste la meilleure introduction aux textes les plus anciens; l'article contient les descriptions détaillées de plusieurs manuscrits. 
Cette insistance sur ce que les chercheurs appellent le dualisme ne s'est pas seulement manifestée dans les manuels d'inquisiteur; elle est présente également dans les documents de la pratique inquisitoriale. Les exemples d'un dualisme classique, « inquisitorial », sont nombreux. Selon la déposition de Raymond Issaura de 1308 dans le registre de Geoffroi d'Ablis, les hérétiques affirmaient l'existence de deux Dieux, un Dieu bon et un Dieu malin ${ }^{29}$. Selon une déposition dans le registre de Jacques Fournier, Pierre Maury a dit qu'il existait deux Dieux, dont le Dieu bon, qui aurait créé les bons esprits, tandis que le monde matériel et les mauvais esprits seraient la création du Dieu mauvais ${ }^{30}$. Des idées très semblables apparaissent aussi dans la déposition de Béatrice de Planissoles, qui, elle, entre dans un dialogue intéressant avec l'inquisiteur en essayant, sans y arriver bien sûr, de nuancer la vision inquisitoriale stéréotypée des choses : interrogée, elle affirme n'avoir jamais entendu les hérétiques parler de deux Dieux, mais les avoir bien entendu dire que c'était le diable qui avait créé ce monde. Ensuite, elle répond à une question savante très révélatrice : non, elle n'a jamais entendu les hérétiques nommer le diable hylè (« la Matière ») $)^{31}$.

29. «Item dicebant, ut dixit, quod erant duo Dii, unus bonus et alius malus et ipsi heretici credebant bono Deo et tenebant fidem suam et illi qui tenent et predicant fidem ecclesie Romane erant de Deo malo ita quod nunquam possunt salvari » (Annette Palès-Gobilliard (éd.), L'inquisiteur Geoffroy d'Ablis et les Cathares du comté de Foix (1308-1309), Paris, 1984, p. 262).

30. «Quod duo erant dii, unus eorum erat « bonesa » et alter « malesa » vel malicia, et, ut dicebat, Deus-bonitas fecerat spiritus et illa que non possunt videri, ita quod bonos angelos et animas et spiritus bonorum hominum fecerat Deusbonitas, set Deus-malitia fecerat omnia corpora alia et omnia que videri possunt vel aliter sentiri, et etiam omnes demones et animas vel spiritus hominum malorum. Et Deus-bonitas regebat et gubernabat celestia, et Deus-malicia regebat et gubernabat istum mundum visibilem et omnia corpora alia. Et Deum-maliciam vocabant Satanam, Deum vero Bonitatem vocabant Deum Claritatis » (J. Duvernoy (éd.), Le Registre d'inquisition de Jacques Fournier, évêque de Pamiers (1318-1325), vol. 2, Toulouse, 1965, p. 179. Cf. la déposition de Pierre Maury lui-même, J. Duvernoy (éd.), Le Registre d'inquisition de Jacques Fournier, évêque de Pamiers (1318-1325), vol. 3, Toulouse, 1965, p. 218).

31. «Interrogata si unquam dictum fuit quod diabolus sic esset principium et factor creaturarum corporalium, quod ipse non esset factus vel productus a Deo vel a quocumque alio, sed esset per se et a se principium, sicut et Deus bonus est principium non factum et productum ab alio omnium spirituum, dixit quod hoc non audivit, nec eciam predicti dixerunt ei quod Deus bonus diabolum fecisset vel non fecisset. Interrogata si unquam audivit a dictis personis quod esset Deus bonus et Deus malus, dixit quod non, sed vocabant Deum illum qui fecerat spiritus, set factorem rerum corporalium diabolum et mundi rectorem, et, ut dixit, non audivit 
Il est intéressant de noter que déjà ces propos, quelque typiques qu'ils soient (et on pourrait continuer à en énumérer d'autres), montrent pourtant plusieurs fissures dans le discours inquisitorial sur le dualisme. C'est le cas du mot interrogata, « interrogée », qui attire l'attention sur les questions de l'inquisiteur, ou du mot ylen (i.e. l'accusatif du mot hylè, « matière »), que Béatrice, aussi admirable qu'elle soit, n'a certainement pas connu : il lui a été suggéré et il trahit manifestement une « manichéisation » inquisitoriale des itinéraires intellectuels dissidents (équation du principe du mal avec la matière). Dans ces cas, les registres disent «trop », en quelque sorte. John $\mathrm{H}$. Arnold a très justement remarqué que ces «surplus de la parole » offrent une possible stratégie de la lecture ${ }^{32}$ : ils permettent de mettre en perspective, de relativiser, mais aussi parfois de confirmer, ce que disent les sources inquisitoriales.

Les fissures du discours inquisitorial deviennent encore plus flagrantes dans le cas d'un type de "marges » du dualisme qu'on pourrait appeler « dualisme des bergers et agriculteurs ». Il s'agit de la croyance selon laquelle ce n'est pas grâce à la volonté de Dieu que croissent les plantes mais grâce à la qualité de la terre ou bien même grâce à l'intervention du diable (1), et de la croyance selon laquelle Dieu n'a pas créé les animaux nuisibles et les tempêtes (2). Les inquisiteurs ont voulu à tout prix assimiler ces propos à leur vision manichéisante de l'hérésie. Or on peut se demander si cette réduction à la croyance en deux dieux est tout à fait justifiée.

\section{LE DUALISME DES BERGERS ET AGRICULTEURS}

La croyance selon laquelle ce n'est pas Dieu qui fait fleurir et grener est présente déjà dans un des plus anciens registres d'inquisition méridionaux, le registre de Bernard de Caux et Jean de SaintPierre (1245-1246). Selon une déposition, une certaine Sabdalena a

eum nominari ylen » (J. Duvernoy (éd.), Le Registre d'inquisition de Jacques Fournier, évêque de Pamiers (1318-1325), vol. 1, Toulouse, 1965, p. 240; J. Duvernoy éd., Le registre d'inquisition de Jacques Fournier, évêque de Pamiers (1318-1325). Corrections, Toulouse, 1972, p. 10).

32. John H. Arnold, Inquisition and Power. Catharism and the Confessing Subject in Medieval Languedoc, Philadelphia, 2001, passim, notamment p. 114-115. 
entendu des hérétiques dire que c'est la terre, et non Dieu, qui fait fleurir et grener ${ }^{33}$.

Ici, ce thème n'est pas lié au dualisme ${ }^{34}$. En revanche, cette association apparaît dans un registre plus tardif - le registre de l'inquisition de Toulouse (1273-1280) ${ }^{35}$. Le lien devient évident dans le registre de Geoffroi d'Ablis, inquisiteur de Carcassonne ${ }^{36}$, qui couvre les années 1308-1309: désormais, cette croyance est associée clairement à des mentions selon lesquelles ce n'est pas Dieu qui a créé le monde ${ }^{37}$. Le registre de Jacques Fournier (1318-1325)

33. «[A]udivit hereticos dicentes quod Deus non faciebat florere nec granare sed terre hoc faciebant per se, et quod matrimonium non valebat, ymmo erat peccatum, sed ipsa testis non credidit predictis erroribus» (J. Duvernoy (éd.), «Le manuscrit 609 de la bibliothèque municipale de Toulouse », http://jean.duvernoy. free.fr/text/pdf/ms609_a.pdf, 2002, p. 166, $\left.\mathrm{f}^{\mathrm{o}} 63^{\mathrm{v}}\right)$.

34. Cf. aussi J. Duvernoy (éd.), « Registre de l'Inquisition de Toulouse (12731280) », http://jean.duvernoy.free.fr/text/pdf/Parnaclatin.pdf, 2003, p. 36 (Paris, Bibliothèque nationale de France, fonds Doat, vol. 25, $\mathrm{f}^{\mathrm{o}} 25^{\mathrm{r}}-25^{\mathrm{v}}$ ) : « [I]nterrogatus si dixit quod de meretricio terre veniebat bladum et vinum et alia bona, respondit quod non tunc. Et ibidem dictus Gausbertus correxit se et recognovit [...]. Item recognovit se dixisse quod pinguedo terre quam ipse vocabat putiam dabat bladum et vinum et alios fructus et bona terre. »

35. «Interrogatus si unquam ipse testis dixit quod Deus nunquam fecit florere nec granare, nec fecit aliqua visibilia, sed putia [ " prostitution»] terre hoc faciebat, et homines faciunt fossata et escolunt terram, dixit quod non. Interrogatus si unquam ipse testis dixit quod duo dii erant, unus bonus et alter malus, dixit quod ipse testis ter vel quater vel pluries dixit in diversis locis quod Garnerius de Cordua, iudex Ruthene, dicebat quod duo dii erant, unus benignus et alter malignus » (J. Duvernoy (éd.), « Registre de l'Inquisition de Toulouse (1273-1280)», p. 125; Paris, Bibliothèque nationale de France, fonds Doat, vol. $\left.25, \mathrm{f}^{\mathrm{o}} 179^{\mathrm{r}}\right)$.

36. Pour un portrait, voir Charles Peytavie, «L'Inquisition de Carcassonne. Geoffroy d'Ablis (1303-1316), le Mal contre le mal », dans L. Albaret (dir.), Les Inquisiteurs. Portraits de défenseurs de la foi en Languedoc (XIII ${ }^{e}-X V^{e}$ siècles), p. $89-100$.

37. « [D]ixit quod audivit eos loquentes de creatione rerum visibilium et dicebant quod Deus non fecerat carnem humanam nec faciebat florere nec granare, ponentes instanciam dicti heretici et dicentes quod si homo poneret granum bladi super lapides, non nasceretur set istud provenit ex putridine terre sicut dicti heretici asserebant» (A. Palès-Gobilliard (éd.), L'Inquisiteur Geoffroy d'Ablis et les Cathares du comté de Foix (1308-1309), p. 104) ou ailleurs : "Item dixit quod audivit a dictis hereticis quod erant duo Dii, unus bonus et alius malus et quod Deus bonus non faciebat florere nec granare nec intromittebat se nisi de spiritibus et quod spitirus [i.e. "spiritus »; err. ed.] seu anime a longo tempore citra sunt create et exeunt de corpore hominis et postea redeunt in corporibus aliorum hominum seu mulierum et dicebant ei quod forte ipsa testis fuerat regina » (ibid., p. 190; cf. p. 134, 148). 
rapporte cette association aussi ${ }^{38}$; quelquefois on en arrive même à l'idée que c'est le Dieu mauvais qui fait fleurir et grener ${ }^{39}$.

On voit donc que la croyance selon laquelle Dieu ne fait pas fleurir et grener se trouve parfois liée à une sorte de dualisme. Mais on ne peut que constater, à la lecture de ces passages, que malgré ce lien occasionnel, elle est différente de la croyance en deux dieux et de la croyance selon laquelle c'est le diable qui a créé le monde. De plus, elle semble résulter plutôt d'une sorte de « rationalisme populaire » que de la prédication des bons hommes. Elle résiste nettement à l'assimilation à l'image inquisitoriale de «l'hérésie manichéenne ».

Cette incompatibilité avec le concept savant du dualisme est encore plus flagrante dans le cas du deuxième thème : la création du loup et des autres animaux nuisibles et des intempéries par le diable. On rencontre cette croyance plus d'une fois dans les registres d'inquisition. Ainsi, Jacques Fournier interrogea Grazide Lizier sur la création des choses matérielles, « parce qu'elle semblait suspecte de l'hérésie manichéenne ». La jeune femme avoua ne pas croire que Dieu a créé les animaux nuisibles; il n'aurait créé que les animaux utiles à l'homme ${ }^{40}$.

38. «Item dicebat ei quod duo erant dii, unus benignus et alter malignus, et quod deus benignus gubernabat et regebat spiritus, et est dominus dictorum bonorum christianorum, id est hereticorum, sed deus malignus regit totum mundum, et omnia corporalia, et est illorum qui sunt subiecti romane Ecclesie et omnium aliorum hominum qui non sunt boni christiani, id est heretici, vel credentes eis, et quod deus bonus non faciebat florere nec granare, sed diligencia et labor hominis faciebat predicta cum bonitate terre » (J. Duvernoy (éd.), Le Registre d'inquisition de Jacques Fournier, évêque de Pamiers (1318-1325), vol. 1, p. 457).

39. «Item dixit quod dictus Guillelmus dicebat ei quod deus benignus non faciebat florere nec granare nec hominum generatione $[\mathrm{m}]$ vel animalium, sed hoc totum faciebat deus malignus » (J. Duvernoy (éd.), Le Registre d'inquisition de Jacques Fournier, évêque de Pamiers (1318-1325), vol. 1, p. 283). $C f$. J. Duvernoy (éd.), Le Registre d'inquisition de Jacques Fournier, évêque de Pamiers (1318-1325), vol. 2, p. 58 : «Et, ut dicebat, ipse non curabat de carne sua, quia ipse nichil habebat in ea, sed erat vermium [ « vermiam» ms., «vermium» corr. ed.]. Pater eciam celestis nichil habebat in carne eius, nec volebat eam habere in regno suo, quia caro humana est domini istius mundi qui eam fecit. Et Pater celestis non vult aliquid habere de illo quod fecit deus et rector huius mundi. Unde et dicebat quod Pater celestis nichil habebat de rebus istius mundi visibilis quod esset suum, nisi solum spiritus quos diabolus eduxit aliquando de celo, ut supra dictum fuit. Et, ut dicebat, Pater celestis nichil omnino faciebat in hoc mundo, nec florere nec granare nec concipere, nec parere, nec fetus producere; et quod generaliter in hoc mundo nichil faciebat. »

40. « [Q]uia suspecta videbatur de heresi manichea, fuit interrogata per dictum dominum episcopum si credebat quod omnia corporalia que in hoc mundo videntur Deus fecisset; respondit quod res corporales bene ad usum humanum et eciam utiles ad [hominem - add. ed.] res illas Deus fecit, sicut homines, bestie quibus 
«Manichéens » certes du point de vue de l'inquisiteur, ces propos ne rencontrent évidemment pas la croyance cathare, selon laquelle tous les corps ont été créés par le diable ou le Dieu mauvais. D'ailleurs, la pensée de la jeune femme telle qu'elle est présentée dans sa déposition forme un système bien cohérent, structuré par la logique de l'utile et de l'agréable; il ne s'agit pas simplement d'une "déformation populaire » des prédications des bons hommes ou de «l'hérésie manichéenne » qu'a soupçonnée Jacques Fournier chez Grazide.

Le cas d'Arnold Cogul est très semblable. Sa pensée ne suit pas les systèmes théologiques cathares. Cogul est plutôt un raisonneur qui a du mal à croire que Dieu aurait créé le loup, puisque les loups avaient l'impudence de dévorer systématiquement ses vaches et brebis - une raison bien compréhensible et surtout peu cathare ${ }^{41}$.

Chez Bernard Franca, on rencontre ce thème dans un cadre plus large. Ce clerc sexagénaire avoue croire depuis quarante ans qu'il y a deux Dieux. Le Dieu bon créa le monde tandis que le Dieu mauvais ruina son œuvre jusqu'à la mission du Christ, destinée à mettre fin au pouvoir du Dieu mauvais. Selon Bernard, c'est le Dieu mauvais qui créa les animaux nuisibles. Pour soutenir l'existence du Dieu mauvais, le clerc cite un psaume évoquant le «Dieu des dieux », Dieu étant le Dieu bon, « dieux » étant une désignation du

homines vescuntur vel veuntur [i.e. " vehuntur»; J. Duvernoy (éd.), Le Registre d'inquisition de Jacques Fournier, évêque de Pamiers (1318-1325). Corrections, p. 12], ut boves, oves, capre, equi, muli, et fructus terre et arborum, quibus homines vescuntur, sed, ut dixit, non credit quod Deus fecerit lupos, muscas, cinsolas, et talia que hominibus sunt nosciva, nec credit eciam quod Deus [J. Duvernoy (éd.), Le Registre d'inquisition de Jacques Fournier, évêque de Pamiers (1318-1325). Corrections, p. 12] Diabolum fecerit, quia res mala est, et nullam rem malam Deus fecit, ut dixit » (J. Duvernoy (éd.), Le Registre d'inquisition de Jacques Fournier, évêque de Pamiers (1318-1325), vol. 1, p. 304.)

41. « [D]ixit et confessus fuit quod a sex annis citra ipse fuit in credencia quod quia Deus est bonus et simplex, in quo nulla malicia est nec esse potest, quod Deus ipse non fecit diabolum vel demones; et quia eciam ipse habebat vacas et oves, et aliquando lupus rapiebat et comedebat de dictis animalibus suis, credidit eciam per dictum tempus quod Deus tam maliciosum animal sicut lupus est non fecisset. Item dixit quod per dictum tempus ipse credidit quod diabolus non fuisset creatus [ « captus » ms., " creatus » corr. ed.] a Deo nec a quocumque alio, sed esset per se ens non factum nec productum. Item dixit quod licet per dictum tempus ipse bene crederet quod unus lupus nunc alium lupum generat, tamen credidit quod a principio primus lupus per se fuit ens, non factus a quocumque alio, sed quod venit in mundum sic, quod nescitur unde venerit vel a quo missus fuerit, sed, ut dixit, credebat et adhuc credit quod omnia alia corpora et spiritus sicut sunt angeli boni, celum, terra, aque, corpora humana et generaliter omnia alia corpora et omnes alias res Deus fecerit » (J. Duvernoy (éd.), Le Registre d'inquisition de Jacques Fournier, évêque de Pamiers (1318-1325), vol. 1, p. 378.) 


\section{Dieu mauvais ${ }^{42}$. Dans une autre déposition, Bernard précise que le Dieu bon ne cause pas les désastres et qu'il n'a pas créé les âmes des mauvais ${ }^{43}$. De nouveau, on rencontre ici un système dualiste}

42. « [D]ixit et confessus fuit sponte quod a toto tempore sue memorie, quod potest esse XL annorum, ut dixit, cum ipse sit sexagenarius vel circa, ipse credidit quod in principio fuerunt duo dii, unus benignus et alter malignus, qui deus malus non fuit factus a deo bono, nec a quocumque alio, sed per se est et fuit, non factus a quocumque alio, quia nec a deo bono, nec a se ipso nec a quocumque alio. Et postea deus bonus fecit mundum et omnes creaturas que in eo sunt, et deus malus, quantum poterat omnia opera dei boni destruebat, cum inimicaretur deo bono, et omnibus creaturis que erant eius, et hoc duravit usque ad Christi incarnacionem. Deinde Christus incarnatus insurgens contra deum malum cepit eum et incathenavit et sic captum detrusit in infernum, ita quod est in tenebris inferni, et ex tunc deus malus amisit suum posse, nec potest ex tunc destruere opera dei boni, nec aliquid aliud facere, licet semper habeat malam voluntatem nocendi, si posset. Et, ut dixit, dictus deus malus de cetero non potuit destruere opera dei boni, et hoc ipse credidit, ut dixit, propter illud quod dicitur in Psalmo: "Deus deorum dominus loqutus est » quia, ut dixit, per « deum » ipse intellexit deum bonum, et per « deorum » ipse intellexit deum malum, et ad hoc eciam ipse adhibebat quoddam exemplum vel istoriam, que talis est: Quedam avis est vocata pellicanus, que est ita clara sicut sol, et sequitur ipsum solem, que avis habuit pullos, et cum illos dimittebat in nido, et ipsa ibat alicubi solem sequendo, veniebat quedam bestia, et dismembrabat dictos pullos, et amputabat eorum rostra; et quando reversus fuerit pellicanus ad suos pullos, inveniens eos dismembratos, et perdidisse rostra, sanabat eos. Et cum hoc frequenter fieret, tandem cogitavit intra se pellicanus, quod claritatem suam absconderet, qua absconsa latitaret iuxta pullos, et cum dicta bestia veniret, caperet et occideret ipsam, ne de cetero eius pullos posset dismembrare vel rostra auferre, quod et factum fuit. Et sic fuerunt liberati pulli pellicani a dismembracione quam faciebat eis dicta bestia, capta per pellicanum bestia ipsa. Et eodem modo, ut dixit, deus bonus fecerat creaturas et deus malus destruebat ipsas, quousque Christus deposuit vel abscondit claritatem suam quando fuit incarnatus ex Maria virgine, et tunc cepit deum malum et posuit eum in tenebris inferni, et ex tunc deus malus non potuit destruere creaturas dei boni. Et propter istam credenciam suam aliquando ipse dixit quod erant duo dii, scilicet deus bonus et deus malus. Item dixit se credere et credidisse toto tempore supradicto quod sicut deus bonus fecit omnes bonas creaturas, sicut sunt angeli, anime humane bone, et corpora, celum, terram, aquas, ignem et aerem, et animalia utilia hominibus, vel ad comedendum vel ad portandum, laborandum, induendum, et pices etiam qui sunt utiles ad comedendum, ita deus malus fecit omnes demones et animalia nociva sicut sunt lupi, serpentes, buffones, musce, et omnia animalia nosciva et venenosa » (J. Duvernoy (éd.), Le Registre d'inquisition de Jacques Fournier, évêque de Pamiers (1318-1325), vol. 1, p. 357-358; J. Duvernoy (éd.), Le Registre d'inquisition de Jacques Fournier, évêque de Pamiers (1318-1325). Corrections, p. 13).

43. «Interrogatus si credebat grandines, fulgura, tempestates, esse factas a deo malo vel a deo bono, respondit se credidisse quod talia fiebant a diabolo vel a deo malo, licet volente vel permittente deo bono, sine cuius permissione vel voluntate deus malus vel diabolus nichil facere poterat, nec eciam potest. [...] Interrogatus quid ipse credebat de animabus [" animalibus » ms., « animabus » corr. ed.] humanis omnibus a quo vel a quibus facte erant, respondit quod ipse credebat quod deus bonus fecisset animas humanas bonorum hominum, et quod deus malus fecisset ani- 
assez complexe qui est autonome et très différent de «l'hérésie manichéenne » définie par les inquisiteurs.

Parmi les dépositions relatant la croyance selon laquelle ce n'est pas Dieu qui créa les animaux nuisibles, celle de Jean Joufre est exceptionnelle par son détail. Joufre dénonce la condamnation d'Arnold Cogul pour ses propos sur la création du loup et il affirme que Dieu bon créa tout, mais pas les démons, les serpents et les loups. Il ne créa pas le diable, puisque tout ce qu'il fait est bon, tandis que le diable est mauvais. Il ne créa pas le serpent, puisque le serpent est venimeux et qu'il a séduit Adam et Ève. Il ne créa pas le loup, puisqu'un loup a égorgé une des tantes du témoin, il est venimeux lui aussi et il fait peur aux bergers ${ }^{44}$.

mas humanas malorum hominum » (J. Duvernoy (éd.), Le Registre d'inquisition de Jacques Fournier, évêque de Pamiers (1318-1325), vol. 1, p. 361).

44. «Item dixit quod anno predicto in quadragesima, postque fuerat factus sermo per dictum dominum episcopum Appamiarum, in quo sermone fuerat condempnatus Arnaldus Cuculli de Lordato ad murum, propter hoc quod inter alios errores dixerat se credidisse quod Deus lupum non fecerat, ipse loquens et Arnaldus Laufredi et quidam alii de quorum nominibus non recordatur erant in platea de Tinhaco, et inter alia dictus Arnaldus Laufredi dixit quod dictus Arnaldus Cuculli fuerat condempnatus ad murum quia dixerat quod Deus non fecerat lupum, quod audiens ipse loquens dixit: "Ita bene ego dicerem coram dicto domino episcopo quod Deus non fecit serpentem nec lupum! » et dictus Arnaldus respondit ei quod si predicta diceret coram dicto episcopo faceret sicut fatuus. Et ipse loquens dixit dicto Arnaldo : «Et quid faceret michi dictus episcopus? », et dictus Arnaldus respondit: "Tu videres!» Deinde post aliquod tempus, et non recordatur de spacio temporis intermedii, ipse et quidam qui est gener Guillelmi de Cornelha [« Conelha » ms., « Cornelha » corr. ed.] de Lordato, qui fuit natus apud Tinhacum, egressi fuerunt de mercato Tarasconis, et revertebantur ad domos suas, et dum ibant simul per vias, quando fuerunt supra pontem Tarasconis vocatum pontem d'Alat, ipse loquens dixit dicto socio suo : "Estne inmuratus Arnaldus Cuculli? », et dictus socius suus respondit ei quod sic, et tunc ipse loquens dixit : «Et posset esse quod fuerit inmuratus dictus Arnaldus quia dicebat quod Deus non fecerat lupum! » et addidit: " Ita bene ego idem dixissem, quia ego non credo quod Deus fecerit serpentem et alia animalia nociva ». Respondit quod ipse credebat quod Deus fecisset omnia que sunt, exceptis demonibus, serpentibus et lupis, quia, ut dixit, de istis non credebat quod Deus fecisset ista, et de serpente propter hoc credebat quod Deus non fecisset eum quia est animal venenosum et etiam quia audiverat dici quod serpens induxerat Evam et Adam quod comederent de fructu ligni de quo Deus prohibuerat quod non comederent [ « comerent» ms., « comederent» corr. ed.], et fecit eum peccare in Deum. De lupo autem credidit quia audiverat dici quod quidam lupus deportaverat per magnum spatium quandam amittam ipsius loquentis et interfecerat eam, et etiam quia est animal venenosum, et dicta animalia magnum timorem incuciunt pastoribus. De diabolo autem hoc credidit quia semper audiverat dici quod diabolus malus est; et credebat quod Deus ita bonus est [quod - add. ed.] res malas [non - add. ed.] fecisset, et in dicta credentia, ut dixit, stetit ab octo annis citra usque modo. Interrogatus quis 
Création des diables, des serpents et des loups par Dieu? Chose impossible.

Quand les inquisiteurs interrogeaient les transgresseurs sur la croyance selon laquelle Dieu n'a pas créé les animaux nuisibles et les intempéries ou bien celle selon laquelle Dieu ne fait pas fleurir et grener, ils le faisaient avec le concept du dualisme « manichéen » en tête. Parfois ces croyances sont même définies explicitement comme des articles de la «secte manichéenne ${ }^{45}$ ». Mais peut-on vraiment assimiler le «dualisme des bergers et agriculteurs » au dualisme manichéisant des inquisiteurs? On a vu qu'il y a de bonnes raisons pour affirmer le contraire. Certes, le « dualisme des bergers et des agriculteurs » se voit rapproché quelquefois du dualisme des inquisiteurs, comme chez le bon homme Guillaume Bélibaste qui a évoqué les intempéries afin de soutenir que ce n'est pas Dieu qui créa le monde visible ${ }^{46}$. Mais ce rapprochement ne semble pas justifier suffisamment une assimilation ou un alignement généalogique des deux pensées. D'abord, la croyance «dualiste » selon laquelle c'est le diable qui fait fleurir et grener n'est qu'un aboutissement d'une autre, d'après laquelle ce n'est pas Dieu qui le fait, mais le travail humain et la qualité de la terre. On cherchera en vain les racines de cette croyance particulière dans les textes dissidents dualistes comme 1'Interrogatio Iohannis ${ }^{47}$ ou le Liber des duobus principiis ${ }^{48}$. C'est dans la pensée concrète des gens qui avaient un contact étroit avec la production agricole que cette idée a pris naissance. Le dualisme n'est ici qu'une couche superficielle et, à en juger par la date tardive des versions à proprement parler dualistes, postérieure. Ensuite, la croyance selon laquelle Dieu n'a pas créé les animaux nuisibles et les

eum docuit, respondit quod nullus, set ipse per se hoc invenit. Interrogatus si ipse aliquem alium docuit, respondit quod non » (J. Duvernoy (éd.), Le Registre d'inquisition de Jacques Fournier, évêque de Pamiers (1318-1325), vol. 2, p. 113-114.)

45. J. Duvernoy (éd.), Le Registre d'inquisition de Jacques Fournier, évêque de Pamiers (1318-1325), vol. 1, p. 304, 355, 378.

46. «Et quia omnia que sunt in hoc mundo visibili, id est celum et terra et omnia que sunt in eis peribunt, et destruentur, ideo nichil istorum fecit, sed, ut dixit, dominus huius mundi fecit omnia ista. Nam, ut dixit, Deus Pater non fecit, nec dixit nisi bonum. «Vides autem quod multa mala sunt in hoc mundo, sicut tempestates, grandines, que non fecit Deus Pater, sed rector huius mundi inimicus Dei Patris » » (J. Duvernoy (éd.), Le Registre d'inquisition de Jacques Fournier, évêque de Pamiers (1318-1325), vol. 2, p. 35-36).

47. E. Bozóky (éd.), Le Livre secret des Cathares. Interrogatio Iohannis, apocryphe d'origine bogomile, Paris, 1980.

48. Christine Thouzellier (éd.), Livre des deux principes. Introduction, texte critique, traduction, notes et index, Paris, 1973. 
intempéries est tout à fait différente du dualisme cathare, réel ou imaginé, où le monde, y compris tous les corps, a été créé par le diable. Enfin, cette croyance telle qu'elle apparait dans le registre d'inquisition de Jacques Fournier a manifestement une autre source que la prédication des bons hommes. Elle est issue de raisonnements autonomes : le Dieu bon n'aurait pas créé ce qui est mauvais; l'existence de deux Dieux est prouvée par un psaume; le loup est un animal venimeux, il fait peur aux bergers et il a égorgé la tante du déposant.

Deux systèmes étiquetés ici "dualisme des bergers et agriculteurs » et « dualisme des inquisiteurs » ne sont pas compatibles, et c'est abusivement que les inquisiteurs les ont assimilés.

\section{Conclusion}

On peut tirer quelques conclusions provisoires de cette enquête :

1) il y avait des idées " dualistes » autonomes qui ne semblent pas être issues de la doctrine cathare telle qu'elle est présentée par les polémistes;

2) il est donc évident que le concept du dualisme est trop vague pour définir, sans d'autres précisions, un mouvement cathare et lui donner une identité

3) afin de maîtriser les discours multiformes qu'ils rencontraient sur le terrain, les inquisiteurs avaient recours à une classification binaire entre «l'hérésie manichéenne », définie notamment par son dualisme, et « la foi que tient et prêche la sainte Église romaine». Cette classification s'est montrée très efficace, certes, mais on a du mal à justifier sa reprise, tellement courante, dans l'historiographie contemporaine*.

Université Masaryk

Faculté des Lettres

Département de l'histoire des religions

Arna Nováka 1

60200 Brno

République tchèque

david.zbiral@post.cz

* Cet article a pu être rédigé grâce à une bourse de recherche de Grantová agentura České republiky (Czech Science Foundation), projet No 401/09/P191 « La terminologie, les classifications et les identités inquisitoriales et leurs résonances dans la tradition historiographique $»$. - J'adresse tous mes remerciements à M. Cédric Giraud (Université Nancy 2) pour ses précieuses remarques. 